\title{
Immunolocalization of Elastase in Human Emphysematous Lungs
}

\author{
V. V. Damiano, A. Tsang, U. Kucich, W. R. Abrains, J. Rosenbloom, P. Kimbel, M. Fallahnejad, and G. Weinbaum \\ Research Division, Department of Medicine, Department of Surgery, The Graduate Hospital,
} and The School of Dental Medicine, University of Pennsylvania, Philadelphia, Pennsylvania 19146

\begin{abstract}
The current working hypothesis concerning the pathogenesis of human pulmonary emphysema proposes that neutrophils migrate through the alveolar interstitium and degranulate, releasing proteolytic enzymes into the interstitium. These enzymes, in particular elastase, can bind to and degrade interstitial elastin. This report describes an immunohistochemical, ultrastructural technique that utilizes polyclonal antibodies to localize neutrophil elastase in human lungs. Using both the immunoperoxidase and the immunogold methods on thin, embedded sections of surgically resected human emphysematous lung tissue, elastase was localized in neutrophils in the lung interstitium and extracellularly in association with interstitial elastic fibers in human lungs that showed local emphysema of varying severity. Quantitative morphometric data were obtained from the lungs of eight patients undergoing lobectomy for removal of pulmonary carcinomas. Patients had preoperative forced expiratory volume $\left(F E V_{1}\right)_{\%}$ levels ranging from 55 to 77 . There was a correlation between a quantitative measure of the local distribution of neutrophil elastase in contact with alveolar interstitial elastin and the local presence of emphysematous change as determined by mean linear intercept of the various histologic sections. These data support the validity of the "protease-protease inhibitor balance hypothesis" as an explanation of the pathogenesis of human pulmonary emphysema.
\end{abstract}

\section{Introduction}

The protease-antiprotease hypothesis of emphysema has recently been reviewed $(1,2)$ and evaluation of the "state-of-the-art" suggests that an elastase can induce emphysema in the lungs of animal models, that alpha-1-protease inhibitor is important in the protection of the lower respiratory tract of humans (3) or animal models (4), and that cigarette smoke may be linked to the development of emphysema because of its ability to stimulate the recruitment of elastase-containing cells to the alveolar interstitium, inactivate elastase inhibitors, and interfere with elastin resynthesis. Thus, the working hypothesis proposes that neutrophils, responding to a chemotactic gradient, can degranulate and release their elastase, which may reach the alveolar interstitium during neutrophil migration. If the elastase is uninhibited by, or protected from, endogenous protease inhibitors, or if it over-

Portions of this work were presented at the 1985 meeting of the American Thoracic Society and have appeared in abstract form (1985. Am. Rev. Respir. Dis. 131[Suppl.]:A386).

Received for publication 12 September 1985 and in revised form 23 April 1986.

J. Clin. Invest.

(c) The American Society for Clinical Investigation, Inc.

$0021-9738 / 86 / 08 / 0482 / 12 \$ 1.00$

Volume 78, August 1986, 482-493 whelms the inhibitor protective screen, the neutrophil elastase binds to and degrades elastin or other connective tissue proteins (5-8). Although circumstantial evidence supports this "proteaseprotease inhibitor balance" hypothesis (9), direct evidence for its role in the pathogenesis of human emphysema is lacking.

This report describes an ultrastructural immunohistochemical method that was used to localize elastase in the lung interstitium. Polyclonal antibodies, against human neutrophil elastase, were applied to thin, embedded sections of human lung prepared for electron microscopy. Using both the immunoperoxidase and the immunogold methods, elastase was localized in the azurophil granules of human peripheral blood neutrophils, in neutrophils present in the lung interstitium, and extracellularly in association with elastic fibers in human emphysematous lungs. Quantitative data obtained from eight patients undergoing surgical lung resection for bronchogenic carcinomas show a correlation between the extent of distribution of neutrophil elastase associated with alveolar interstitial elastin and the mean linear intercept (MLI) ${ }^{1}$ of the resected lung tissue. This method provides information regarding the relative amount and the distribution of elastase in the lung. It contributes significant evidence in support of the "balance" hypothesis as a contributing factor in our understanding of the pathogenesis of human emphysema.

\section{Methods}

\section{Preparation of lung tissue}

Human lung tissue was obtained, after informed consent, from eight patients undergoing lobectomy for pulmonary carcinoma. Age, smoking history, and involved lobe for each patient are shown in Table I. The ages of the patients ranged from $\mathbf{4 0}$ to $\mathbf{7 6} \mathrm{yr}$, and right or left upper or lower lobes from both male and female patients were studied. Smoking history showed a wide range while preoperative spirometry (Table II) demonstrated values of forced expiratory volume $\left(\mathrm{FEV}_{1}\right)_{0}=(\mathrm{FEV} /$ forced vital capacity [FVC] $\times 100$ ranging from 55 to 77 .

The resected lobes from each patient were fixed for $24 \mathrm{~h}$ by instillation of $4 \%$ formaldehyde into the airways at constant transpulmonary pressure of $25 \mathrm{~cm}$ water. After fixation, each lobe was cut sagitally into six slices and one tissue sample was randomly selected from each lung slice. Histologic slides were prepared from each of the tissue specimens. Four of the six histologic slides that exhibited well-inflated parenchymal tissue and were free of atalectasis, larger bronchioles, or major blood vessels were used to determine the subjective microscopic estimate and quantitative index of the severity of emphysema. 10 fields from each histologic slide were individually scored for the severity of emphysema using scores of 1,2 , or 3 for mild, moderate, or severe emphysema, respectively, and the value of emphysema severity most frequently observed was determined for each slide. In addition, MLI was also determined as a quantitative index of emphysema severity for each of the histologic slides (10).

The remaining portions of each of the tissue samples from which the histologic sections were taken were prepared for electron microscopic

1. Abbreviations used in this paper: FEV, forced expiratory volume; FVC, forced vital capacity; MLI, mean linear intercept; PAP, peroxidase/antiperoxidase; \% EPGS, percentage of elastase-positive grid squares; alpha1-PI, alpha-1-proteinase inhibitor. 
Table I. Description of Patient Population

\begin{tabular}{lllc}
\hline Patient number & Age (sex) & Involved lobe & Smoking history \\
\hline & & & pack-yr \\
1 & $62(\mathrm{M})$ & LUL & 25 \\
2 & $67(\mathrm{M})$ & RUL & 15 \\
3 & $76(\mathrm{~F})$ & RUL & 60 \\
4 & $40(\mathrm{M})$ & LLL & 40 \\
5 & $77(\mathrm{~F})$ & LUL & 100 \\
6 & $67(\mathrm{~F})$ & RUL & 60 \\
7 & $69(\mathrm{~F})$ & RUL & NS \\
8 & $52(\mathrm{M})$ & LLL & 48
\end{tabular}

studies by further sectioning into smaller blocks $\left(1 \mathrm{~mm}^{3}\right)$ and fixation for $12 \mathrm{~h}$ in $0.5 \%$ glutaraldehyde in a modified Millonig's phosphate buffer (11) balanced to an osmolality of $330 \mathrm{mosmol}$ and then fixed for $30 \mathrm{~min}$ in $1 \% \mathrm{OsO}_{4}$ in $0.2 \mathrm{M}$ sym-collidine, $\mathrm{pH} 7.4$. The specimens were rinsed in water and dehydrated in a series of graded ethanol solutions, cleared with propylene oxide, and embedded in Epon.

\section{Preparation of neutrophils}

Human white blood cells, obtained from healthy volunteers, were fractionated using a Percoll gradient $(12,13)$. Approximately $90 \%$ of the cells isolated were neutrophils. Viability was $95-98 \%$ as judged by the ability of the neutrophils to exclude $0.125 \%$ Trypan blue. The isolated neutrophils were prepared for electron microscopy by fixation of the cells in $\mathbf{0 . 5 \%}$ glutaraldehyde in 330 mosmol Millonig's phosphate buffer (11). The neutrophils were then washed several times with Tris buffer, $\mathrm{pH} 7.4$, by centrifugation at $700 \mathrm{~g}$ for $10 \mathrm{~min}$, resuspended, postfixed with $1 \% \mathrm{OsO}_{4}$ buffered in $0.2 \mathrm{M}$ sym-collidine, $\mathrm{pH} 7.4$, pelleted by centrifugation, washed, dehydrated, and embedded in Epon.

\section{Sample sectioning}

The Epon blocks were trimmed and thick-sectioned $(2 \mu \mathrm{m})$ with the LKB Pyramitome (LKB Instruments, Inc., Gaithersburg, MD) 11800 (14). These sections were stained with $1 \%$ toluidine blue in $1 \%$ sodium borate and the neutrophils or areas of parenchymal lung tissue were selected for thin sectioning by light microscopic inspection of the $2-\mu \mathrm{m}$ thick sections. Three serial thin sections $(60 \mathrm{~nm}$ thick), measuring 0.5 $\times 0.7 \mathrm{~mm}$, were cut from each of the selected blocks. The sections, covering approximately 30 grid squares on 300 -mesh nickel grids, were immunologically stained with the appropriate antibody reagents as described below.

\section{Antibody preparation}

Human neutrophil elastase was purified according to published procedures (15-17) that involve isolation of neutrophils, separation of lysosomal granules by differential centrifugation and extraction, and purification of elastase by gel filtration and affinity chromatography. Antiserum was prepared in rabbits by multisite subcutaneous injections of $50 \mu \mathrm{g}$ of purified human neutrophil elastase in complete Freund's adjuvant. Subsequent booster injections, $\sim 2 \mathrm{wk}$ apart, contained $25 \mu \mathrm{g}$ of the enzyme in incomplete Freund's. At least three to four injections were required to achieve a sufficiently high antibody titer. A week after the last injection, the animals were bled by ear venipuncture and serum was collected by centrifugation. The IgG fraction was obtained by precipitation with $35 \%$ ammonium sulfate and anion exchange chromatography in $0.025 \mathrm{M}$ phosphate buffer, $\mathrm{pH}$ 6.7. The dialyzed IgG fraction was stored at $4^{\circ} \mathrm{C}$ in the presence of $0.02 \% \mathrm{NaN}_{3}$ as a preservative. The specificity of the antielastase IgG was tested by both immunodiffusion and immunoelectrophoresis (18). Both methods yielded a single line of precipitation against either purified human neutrophil elastase or the crude neutrophil extract. Prior to using the anti-human neutrophil elastase $\mathrm{IgG}$, the titer and specificity were measured by an enzyme-linked immunosorbent assay (ELISA) similar to a previously reported procedure (19).

\section{Preparation of purified amorphous human lung elastin}

The amorphous component of human lung elastin was prepared according to published procedures (8). Prior to use for electron microscopic studies, the amorphous elastin material was ground finely in a ball mill under liquid nitrogen until it passed through a 400-mesh screen. Weighed pieces of purified lung elastin were exposed to two concentrations of human neutrophil elastase ( $2.5 \mu \mathrm{g}$ of elastase/mg dry wt elastin and 25 $\mu \mathrm{g}$ of elastase/mg dry wt elastin). The elastase-treated elastin powder was then fixed in $0.5 \%$ glutaraldehyde for $1 \mathrm{hr}$, embedded, and sectioned for electron microscopy studies.

\section{Immunologic staining}

Both the immunoperoxidase (20) and the immunogold methods (21) were used to localize elastase in thin sections prepared for electron microscopy. The initial step in both of these procedures involved incubation of the thin sections on the nickel grids in $0.5 \%$ bovine serum albumin (BSA) in $0.05 \mathrm{M}$ Tris-saline, $\mathrm{pH} 7.6$, for $10 \mathrm{~min}$ to block the nonspecific binding sites on the section surface. The sequence of steps listed below were then followed.

Immunoperoxidase method. Thin sections of neutrophils or lung tissue on nickel grids were incubated in:

(a) Specific rabbit anti-human neutrophil elastase IgG or, for controls, preimmune IgG or specific rabbit anti-human neutrophil elastase IgG preadsorbed with excess antigen. The antibody solutions were diluted 1:100 with $0.5 \% \mathrm{BSA}$ in $0.05 \mathrm{M}$ Tris-saline, $\mathrm{pH}$ 7.6 (Tris), and incubated

Table II. Pulmonary Function of Patient Population

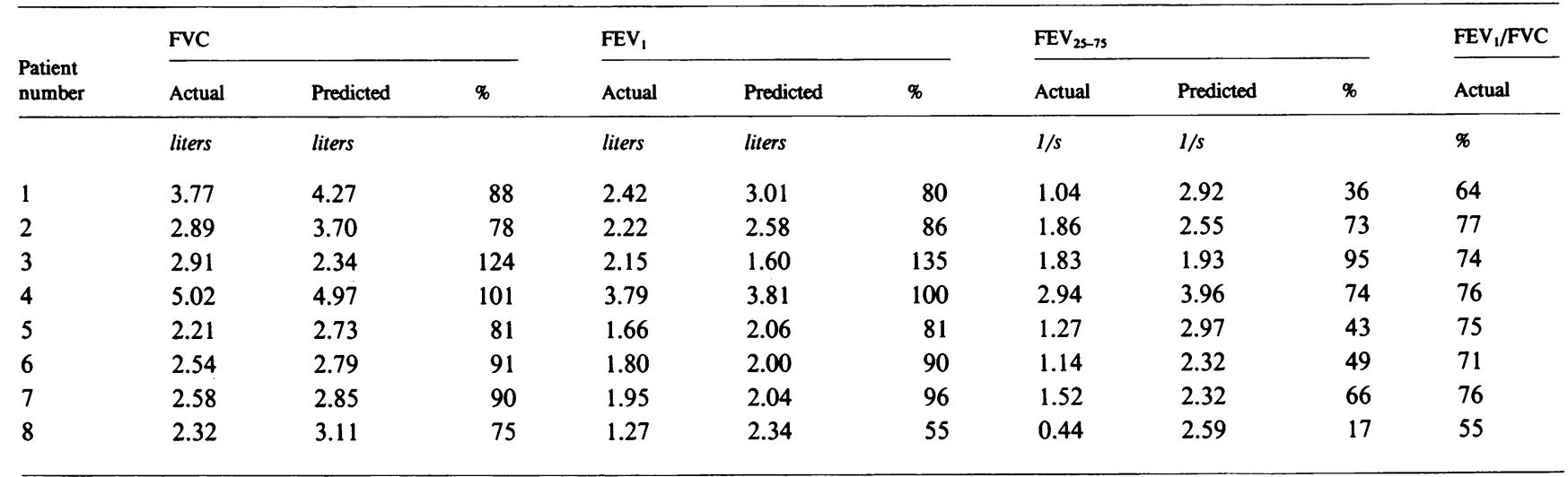


on the thin sections at $4^{\circ} \mathrm{C}$ for $72 \mathrm{~h}$. All subsequent exposures were conducted at room temperature.

(b) Three rinses, each 5-10 min, in Tris.

(c) Goat anti-rabbit IgG (Cappel Labs, Malvern, PA) diluted 1:100 in Tris containing $0.5 \%$ BSA for $30 \mathrm{~min}$.

(d) Three rinses in Tris, each 5-10 min.

(e) Peroxidase/antiperoxidase (PAP) prepared in rabbit (Sternberger Meyer Immunocytochemicals, Inc., Jarrettsville, MD) diluted 1:50 with $0.5 \%$ BSA in Tris for $30 \mathrm{~min}$.

$(f)$ Hydrogen peroxide $(0.00125 \%)+$ diaminobenzidine tetrahydrochloride $(0.12 \%)$ in Tris for $5 \mathrm{~min}$.

(g) Three rinses in water, each 5-10 min. (h) $1 \% \mathrm{OsO}_{4}$ in water for $15 \mathrm{~min}$.

(i) One rinse in water.

$(j)$ Counterstain in $1 \%$ uranyl acetate in water at $60^{\circ} \mathrm{C}$ for $3 \mathrm{~min}$ and lead citrate for $5 \mathrm{~min}$ at room temperature.

Immunogold method. The immunogold method included the first two steps described above for the immunoperoxidase method except that a dilution of 1:1,000 was used for rabbit anti-human neutrophil elastase IgG. The sections were then exposed to gold-conjugated goat anti-rabbit IgG (Structure Probe Inc., West Chester, PA) diluted 1:5 in Tris containing $0.5 \%$ BSA for $30 \mathrm{~min}$. This was followed by three rinses in water and counterstaining with $1 \%$ uranyl acetate in water at $60^{\circ} \mathrm{C}$ for $3 \mathrm{~min}$ and lead citrate for $5 \mathrm{~min}$ at room temperature.

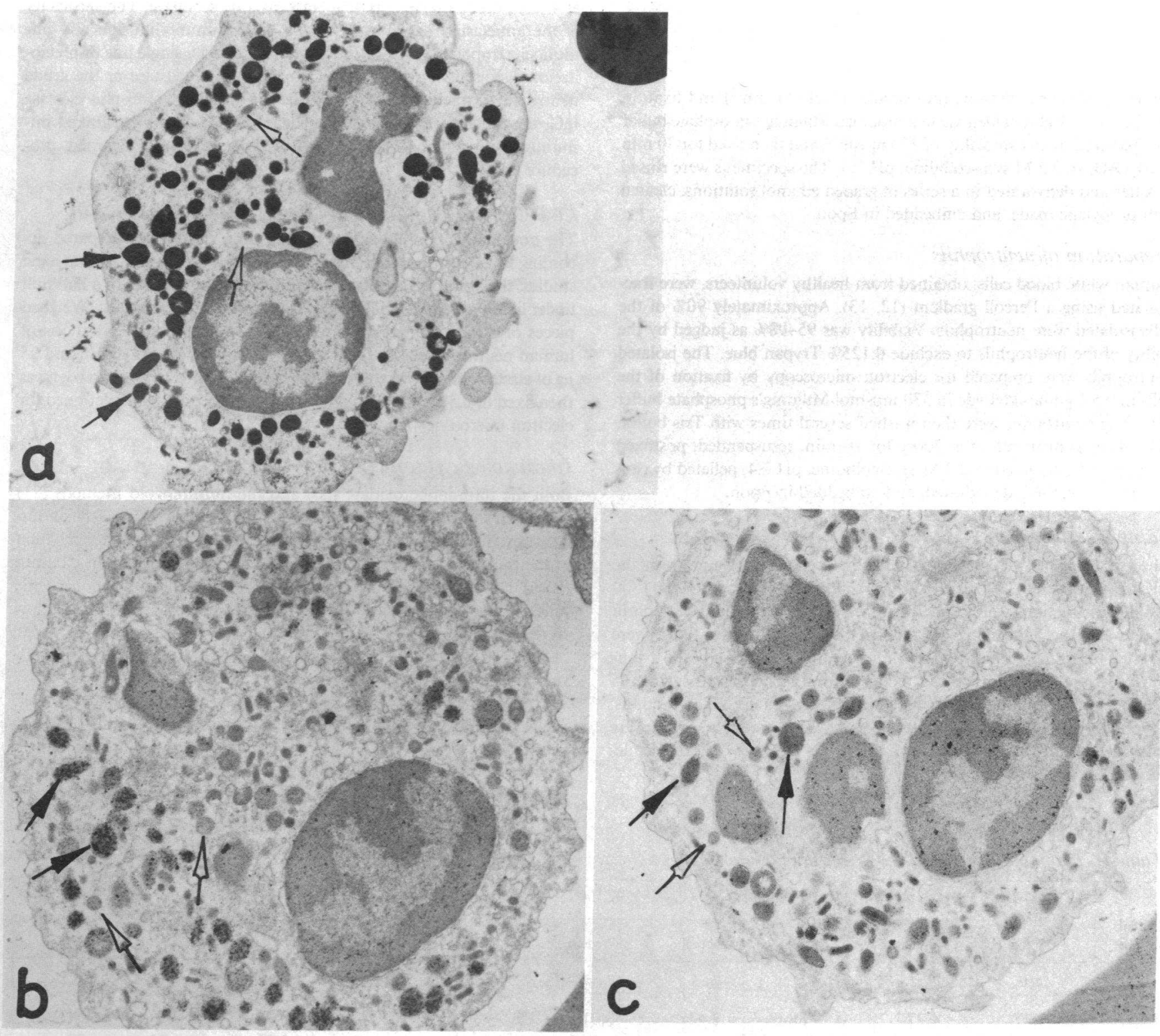

Figure 1. (a) Electron micrograph of a human blood neutrophil showing the histochemical localization of endogenous peroxidase in large dark-contrasted azurophil granules (solid arrows) using the Graham and Karnovsky (24) method. Open arrows identify low-contrasted peroxidase-free specific granules. Uranyl acetate + lead citrate counterstain. $\times 8,140$. (b) Section treated with rabbit antielastase IgG in the immunohistochemical procedure to localize elastase. Positive elastase reaction seen at the solid arrows on the azurophil granules. Negative reaction on the smaller specific granules at the open arrows. Uranyl acetate + lead citrate stain. $\times 8,130$. (c) Electron micrograph of human neutrophil. Endogenous peroxidase was not revealed when preimmune rabbit IgG was used instead of the rabbit antielastase IgG in the immunohistochemical staining procedure of the thin sections. Large azurophil granules marked by the solid arrows have similar contrast as the smaller specific granules marked by open arrows. Uranyl acetate + lead citrate counterstain. $\times 8,130$. 


\section{Quantitation of elastase distribution}

Peroxidase method. Thin sections stained using the immunoperoxidase method were examined at approximately $\times 8000$ magnification in the Jeol 100 CX transmission electron microscope (JEOL USA, Peabody, MA) at $80 \mathrm{kV}$. The electron microscope grid served as a sampling grid and each grid square containing lung tissue was examined to determine whether there was a peroxidase-positive reaction for elastase observed in association with interstitial elastin. The number of grid squares containing elastin and exhibiting a positive elastase reaction and the total number of grid squares containing elastin in the alveolar interstitium were determined for each of the sections. MLI was determined independently, by light microscopy, for each of the histologic slides. The fraction of elastase-positive grid squares expressed as a percent of total elastincontaining grid squares was correlated to the severity of emphysema by the method of least squares. Using linear regression analysis, an estimating equation of the form $Y=a+b X$ was obtained, where $Y$ is the percent of elastase-positive grid squares and $X$ is the mean linear intercept. The relationship between the distribution of elastase and the mean linear intercept was determined from the coefficient of correlation.

Gold method. Thin sections from each patient lung, immunologically stained using the immunogold method, were examined in the electron microscope and electron micrographs of the elastin in each grid square were taken. The number of gold particles was counted and the area of elastin on which the gold particles were observed was measured in square micrometers. The density of gold particles per square micrometer of elastin was determined for each of the micrographs and an average value of gold particle density on elastin in the lung parenchyma of the four samples from each patient was recorded. Gold particle densities on purified amorphous elastin and on elastin treated with elastase were also determined. For controls, antibody against elastase preadsorbed with an excess of antigen bound to an insoluble matrix was used on both the purified amorphous lung elastin and the experimental lung sections.

\section{Results}

Localization of elastase in neutrophils. The human neutrophil is known to contain at least two populations of granules (azurophil and specific) (22). The azurophil granules, a rich source of elastase (23), served as a good standard for the immunolocalization of elastase. The Graham and Karnovsky (24) technique, which is applied prior to dehydration and embedding, was used to identify the azurophil granules in the neutrophil by localizing the endogenous peroxidase (an azurophil granule marker) as seen in Fig. $1 a$. The azurophil granules (dark contrast, solid arrows) are larger $(0.3 \mu \mathrm{m})$ than the specific granules $(0.1$ $\mu \mathrm{m}$, low contrast, open arrows). Serial sections of a single human neutrophil treated with specific rabbit anti-human neutrophil elastase IgG or the preimmune rabbit IgG in the immunoperoxidase procedure are shown in Fig. $1 b$ and $c$, respectively. A positive reaction for elastase was observed in the large azurophil granules of the neutrophils when anti-human neutrophil elastase IgG was used as the primary antibody as seen in Fig. $1 b$ (solid arrows) and a negative reaction in the smaller specific granules at the open arrows (Fig. 1 b). A negative reaction was observed for both the azurophil granules (solid arrows) and the specific granules (open arrows) for the controls treated with the preim-

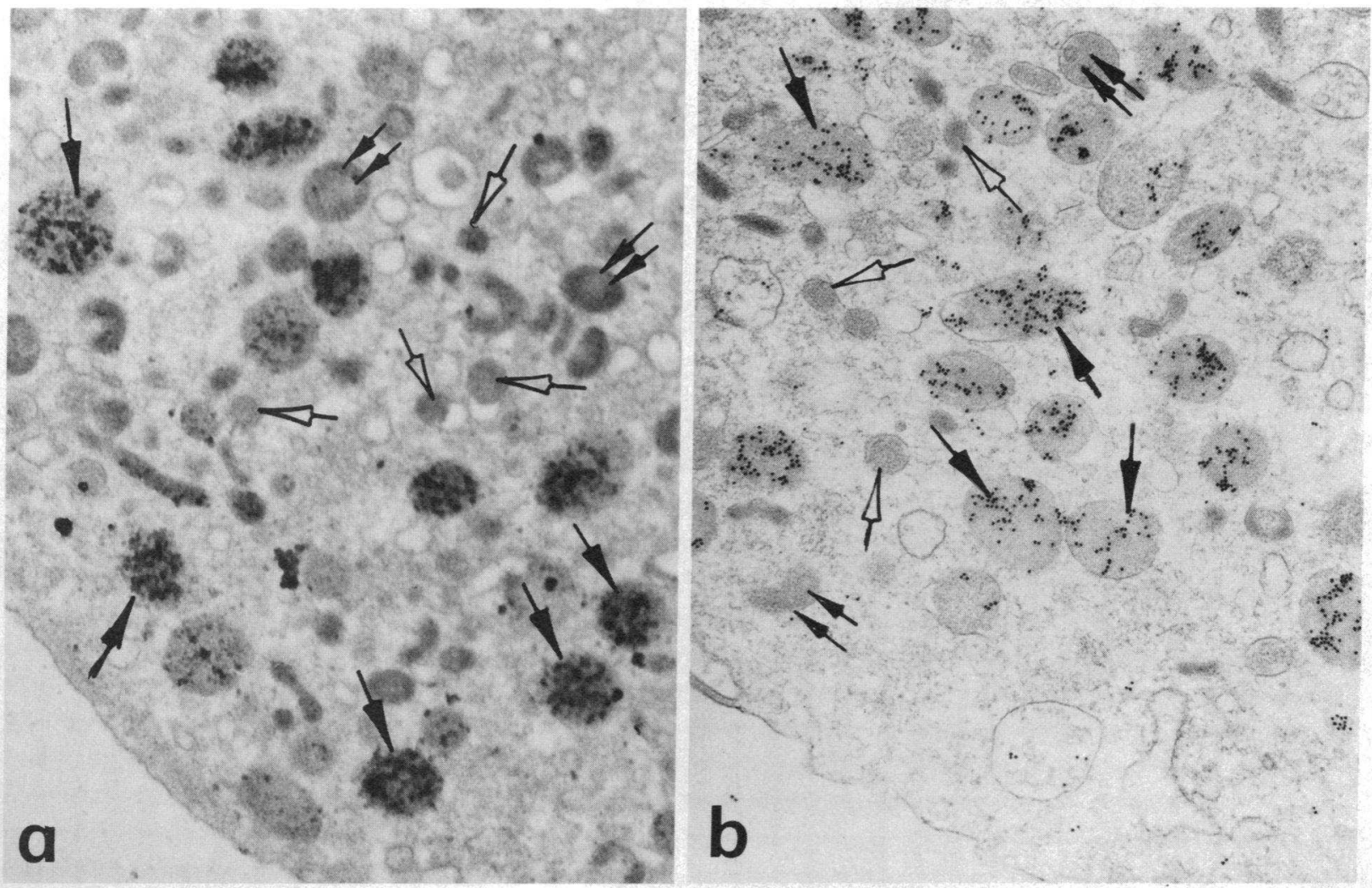

Figure 2. Electron micrograph of a portion of a human blood neutrophil. (a) Immunoperoxidase staining shows elastase-positive reaction product on the large azurophil granules at the solid arrows. Smaller specific granules at open arrows are elastase-negative. Intermediatesize granules at double arrows also negative. (b) Immunogold localiza- tion of elastase. Positive elastase reaction on the large azurophil granules at solid arrows. Negative reaction to elastase on smaller specific granules. Intermediate size granules at double arrows also elastase-negative. Uranyl acetate + lead citrate counterstain. $\times 20,000$. 
mune rabbit IgG (Fig. $1 c$ ). In addition, the endogenous peroxidase, seen in Fig. $1 a$, was not revealed in the azurophil granules when the primary, rabbit anti-human neutrophil elastase, antibody was omitted in the immunoperoxidase staining of the sections or when the sections were only treated with hydrogen peroxide and diaminobenzidine as shown in steps $f-j$ in the immunoperoxidase method. A similar negative reaction was observed for the controls treated with rabbit anti-human elastase IgG preadsorbed with an excess of antigen. These results show that endogenous peroxidase is inactivated during the embedding procedure and its activity does not cause a false "elastase-positive" reaction. At higher power, in Fig. $2 a$, a much clearer distinction between the largest elastase-positive granules (solid arrows) and the smallest elastase-negative granules (open arrows) can be made. Some intermediate-sized granules (double arrows), were also elastase-negative. Similar results were observed with the immunogold method, as seen in Fig. $2 b$. An elastase-positive reaction was observed with accumulation of colloidal gold particles on the azurophil granules (solid arrows) while a negative reaction was observed on the specific granules (open arrows). Some intermediate-sized granules were also elastase-negative in the immunogold procedure (double arrows, Fig. $2 b$ ). Thus, the immunogold method corroborated the immunoperoxidase method and offered further proof that the immunologic method localized elastase and not endogenous peroxidase within the azurophil granules. As expected, some nonspecific binding was observed on the nuclei, cytoplasm, and Epon. Quantitation of the average gold particle density on the azurophil granules was $\sim 50$ times that observed for the average nonspecific background on the nuclei, cytoplasm, and Epon.

Localization of elastase in the alveolar interstitium. Using the method described above for peripheral blood neutrophils, elastase was identified both within neutrophils (Fig. 3) and extracellularly in the interstitium of alveolar walls. Immunogold localization of elastase within the largest granules of the interstitial neutrophil and extracellularly in association with elastin is demonstrated in Fig. 3 at the solid arrows and open arrows, respectively. Both the immunoperoxidase (Fig. $4 a$ ) and immunogold (Fig. $4 b$ ) methods showed localization of extracellular neutrophil elastase in association with interstitial elastin. A positive reaction to elastase is demonstrated by the electron-dense peroxidase reaction product in Fig. $4 a$ and by the accumulation of colloidal gold particles in Fig. $4 b$ at the solid arrows in association with the elastin $(E)$. Little or no elastase-positive reaction was found in association with basement membrane $(B M)$ or with collagen $(C)$ in Fig. $4 \mathrm{~b}$. The level of the gold particle density measured on tissue components other than elastin was no greater than the background level. Similarly, macrophages which were only occasionally observed in these lung sections did not exhibit concentrations of gold particles above the back-

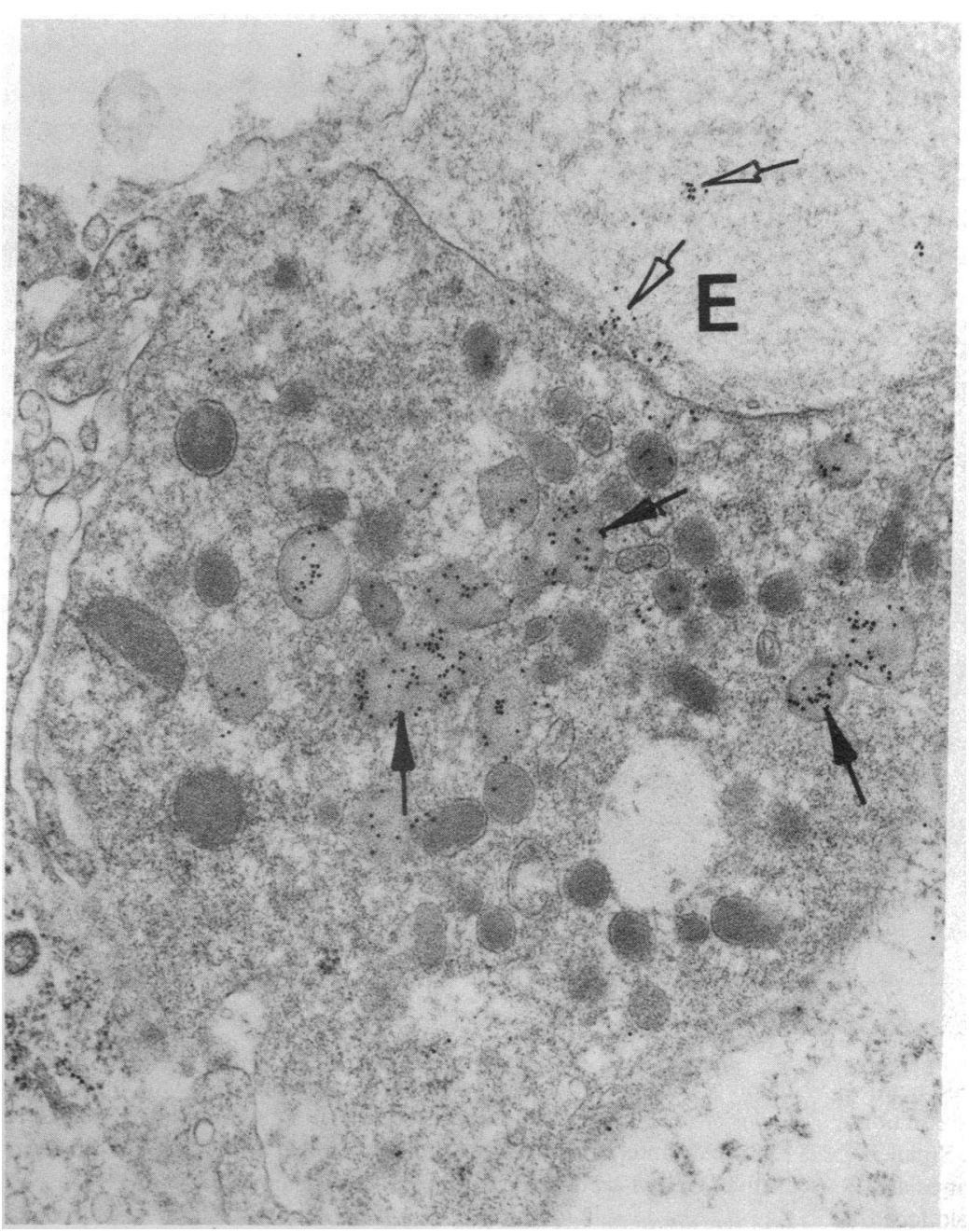

Figure 3. Electron micrograph of section of lung from patient 4 showing the immunogold localization of elastase on the azurophil granules of an interstitial neutrophil at solid arrows. Elastase-positive reaction at open arrows on extracellular elastin $(E)$. Uranyl acetate + lead citrate counterstain. $\times 30,000$. 

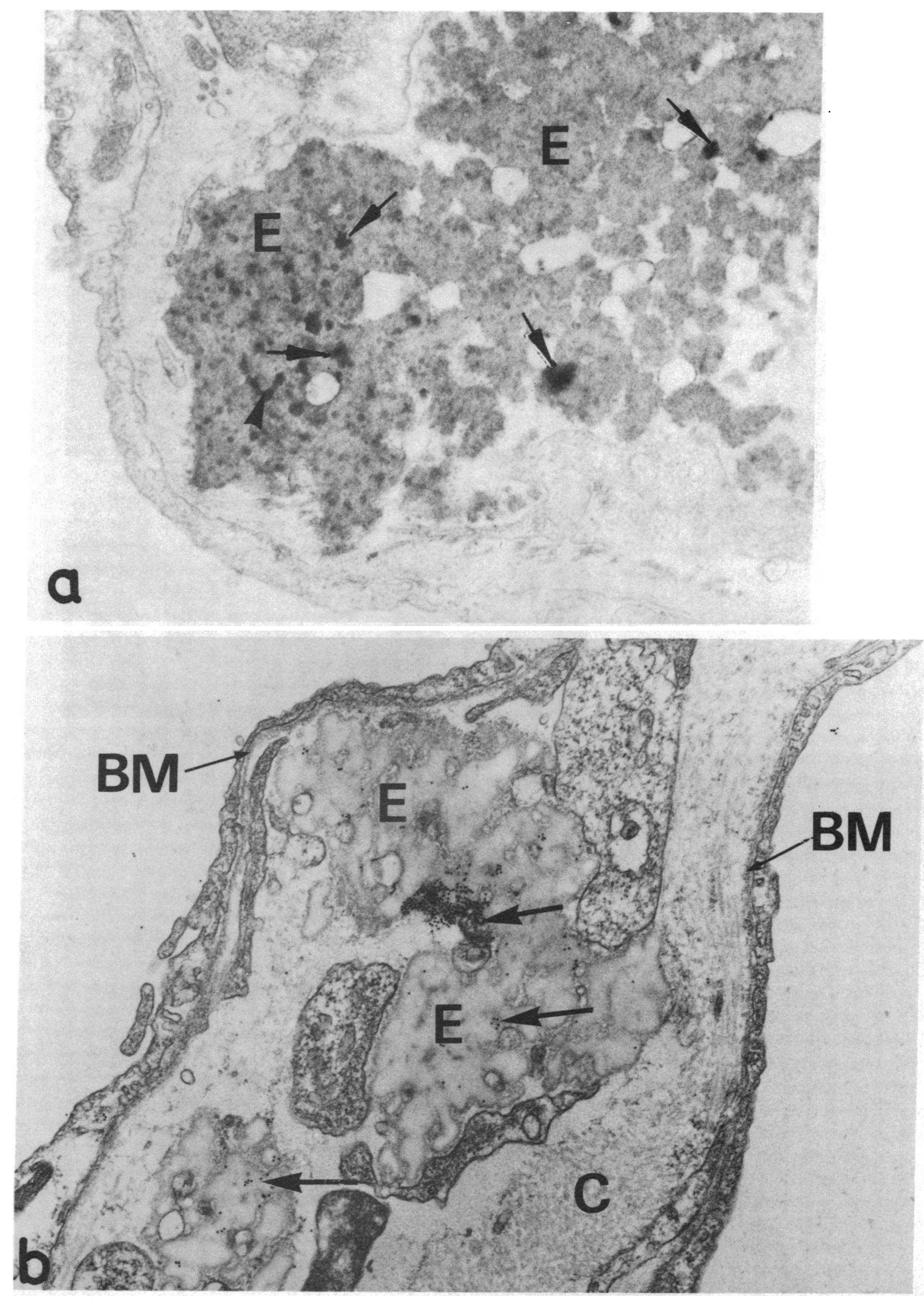

Figure 4. Electron micrographs of portion of alveolar wall from patient 5 showing immunohistochemical localization of elastase on elastin. (a) Immunoperoxidase stain shows electron-dense elastase-positive reaction product on elastic fiber $(E)$ at the solid arrows. $\times 45,000$. (b)
Immunogold stain shows concentrations of colloidal gold particles on elastic fiber $(E)$ at solid arrows. Collagen $(C)$ and basement membrane $(B M)$ show little or no colloidal gold binding. Uranyl acetate + lead citrate stain. $\times 29,000$. 

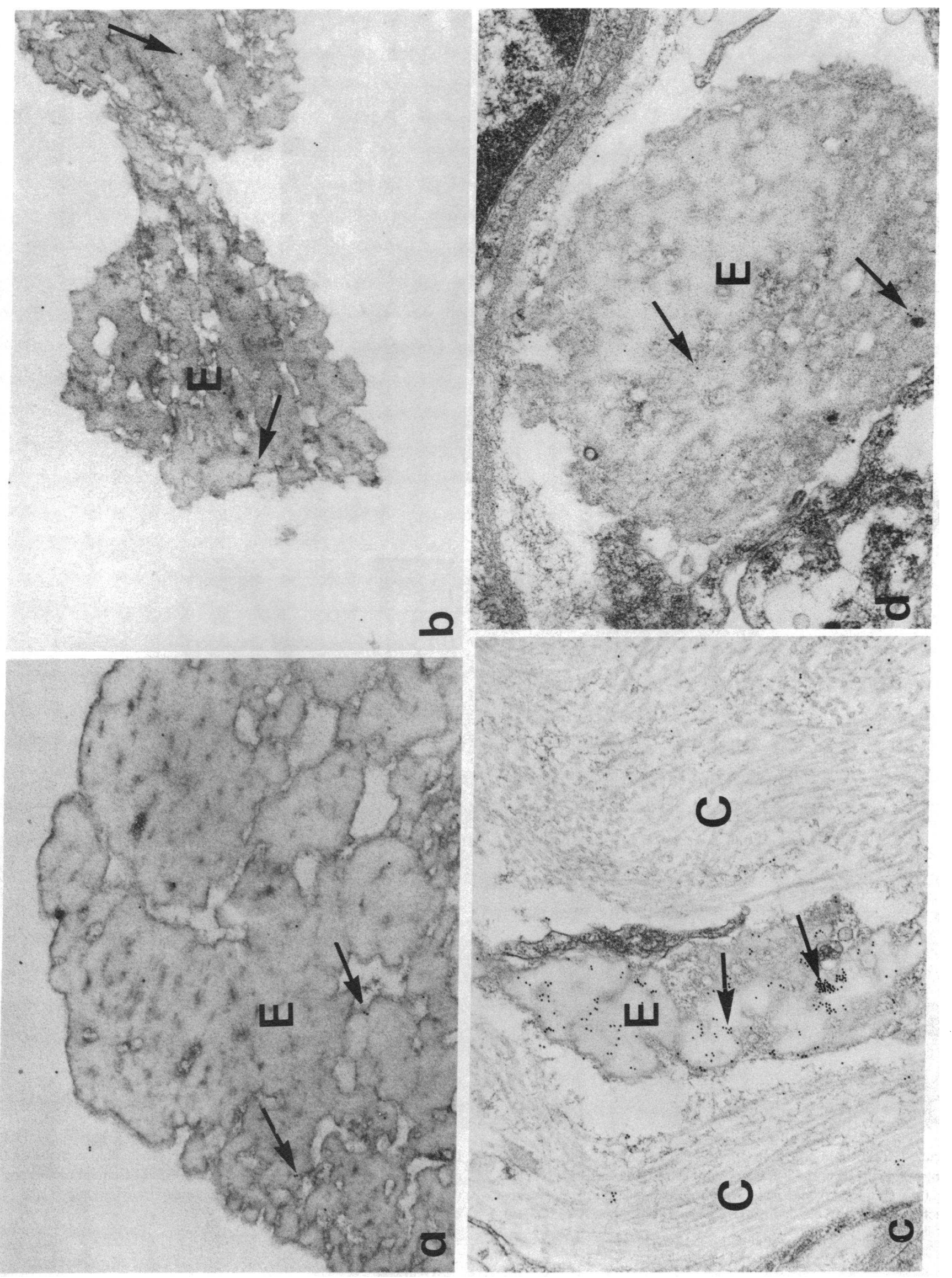
ground levels. The distribution of elastase on elastin was patchy. Regions without elastase could be found in all of the lung sections examined. Some sections from mild emphysematous lungs, rated subjectively as 1 , were completely free of elastase-positive reaction product and exhibited little or no gold binding, whereas sections from the severely emphysematous lungs, rated subjectively as 3, exhibited an abundance of reaction product and high concentrations of gold associated with elastin.

The immunohistochemical data do not establish the enzymatic activity of the neutrophil elastase which is immunolabeled. The interaction between alpha-1-proteinase inhibitor (PI) and elastase is essentially irreversible (25) and once complexed, the enzyme/inhibitor complex is recognized by the antibody (19). However, the complexed elastase does not selectively bind to elastin. This was established in earlier studies when elastase was purified on elastin affinity columns (8). Elastase inhibited by alpha-1-PI could not be purified in this manner in that it did not adhere to the column (data not shown). As can be seen in Figs. 3 and 4, the immunolocalization of elastase is enriched on the interstitial elastin. This suggests that active enzyme attached to insoluble substrate is the antigen being identified since inactive enzyme does not bind to elastin.

Localization of elastase on elastin. Purified amorphous lung elastin was used as a negative elastase control as seen in Fig. 5 $a$. Little or no gold binding (four gold particles $/ \mu \mathrm{m}^{2}$ elastin) was observed using the specific rabbit antielastase as the primary antibody. Similarly, purified amorphous lung elastin treated with rabbit antielastase antibody preadsorbed with antigen showed little or no gold binding (six gold particles $/ \mu \mathrm{m}^{2}$ elastin) as seen in Fig. $5 b$. A comparison of the binding of gold to elastin on a lung section immunologically stained using the specific antibody and a lung section immunologically stained using the preadsorbed antibody is shown in Fig. $5 c$ and $d$, respectively, using lung sections from patient 6 (severe emphysema). Using the specific antibody, a gold count of 155 gold particles $/ \mu \mathrm{m}^{2}$ of elastin, above background, was observed while the control treated with preadsorbed antibody had a gold concentration of six gold particles $/ \mu \mathrm{m}^{2}$ of elastin. Sections of human lung elastin treated with human neutrophil elastase $(2.5 \mu \mathrm{g} / \mathrm{mg}$ and $25 \mu \mathrm{g} / \mathrm{mg}$ dry wt elastin) exhibited a gold concentration of 120 and 1,420 particles/ $\mu \mathrm{m}^{2}$, respectively, as seen in Fig. $6 a$ and $b$. Again, controls using preadsorbed antibody showed gold particle levels of less than 10 gold particles $/ \mu \mathrm{m}^{2}$ (figure not shown).

Quantitation of elastase distribution in lung tissue. Lung specimens from eight patients were studied in detail. Subjective evaluation of lung microscopic sections for emphysema severity utilized three classifications according to the typical appearances, as shown in Fig. $7 a, b$, and $c$. Mild microscopic emphysema showed alveoli of uniform size with an occasional enlarged air space and minimal alveolar wall destruction. Moderate emphysema was identified by an increased number of enlarged air spaces, as marked by the asterisks. Severe emphysema showed marked nonuniformity of alveolar size, a greater number of en- larged air spaces and an abundance of alveolar wall destruction. This microscopic emphysema was patchy and did not necessarily relate to the overall clinical status of the patients.

Table III summarizes the averages of the histologic and immunolocalization data obtained from the eight lobes studied. The histologic severity and MLI were determined from 10 areas of each of four slides from different sections of the respective lobes. Although six slides were originally prepared for each lobe, only four were used because of atelectasis or the presence of an excessive number of large airways or major blood vessels. The data in Table III show that two lobes in group I (mild emphysema), showed $6 \%$ and $9 \%$, respectively, of the grid squares containing elastin to be elastase-positive and 14 and 16 gold particles/ $\mu \mathrm{m}^{2}$ of elastin. Three lobes in group II (moderate emphysema), showed more of the grid squares containing elastin to be elastasepositive and to have higher gold particle density. Groups I and II were significantly different at the $P<0.005$ level for PAP distribution. Three lobes in group III (severe emphysema), showed $35 \%, 56 \%$, and $54 \%$, respectively, of the grid squares containing elastin to be elastase-positive and 26,87 , and 70 gold particles $/ \mu \mathrm{m}^{2}$ elastin. Groups II and III were significantly different at $P<0.05$ for PAP and $P<0.001$ for gold distribution. Groups I and III were significantly different at $P<0.005$ level for PAP and $<0.001$ for gold. A comparison of average values of gold particle density or percent elastase-positive grid squares with corresponding average MLI values show linear correlation coefficients of 0.71 and 0.91 , respectively (Table III).

A more objective correlation of the distribution of elastase on elastin with the severity of emphysema was obtained by evaluation of each of the electron microscopic grids for percent elastase-positive grid squares and each of the histologic slides for the average MLI. A plot of the percentage of elastase-positive grid squares containing elastin vs. the average MLI from each of four histologic lung specimens from each of the eight patients is shown in Fig. 8.

These results show a significant increase in the distribution of extracellular, interstitial neutrophil elastase with increasing severity of emphysema, as measured by MLI. The linear regression line and estimating equation correlating the percentage of elastase-positive grid squares (\% EPGS) to MLI (mm) is given by: $\%$ EPGS $=-44+216 \mathrm{MLI}$, with a correlation coefficient of $r=0.81$.

\section{Discussion}

The immunologic ultrastructural localization of elastase in both human neutrophils and extracellularly in the interstitium of alveolar walls of human lungs offers a direct method to relate the presence and site of elastase binding with emphysematous changes in lung tissue. The results presented here demonstrated that elastase can be localized within the azurophil granules of neutrophils and in the lung, primarily in association with interstitial elastin, using either the immunoperoxidase or the im-
Figure 5. (Opposite) Electron micrographs of (a) purified human amorphous lung elastin. Immunogold stain using rabbit anti-human neutrophil elastase IgG as primary antibody. Gold at solid arrows. Gold density on elastin $=$ four particles $/ \mu \mathrm{m}^{2}$. (b) Purified human amorphous lung elastin treated as control with rabbit anti-human neutrophil elastase IgG preadsorbed with excess antigen. Gold at solid arrows. Gold density on elastin $=$ six particles $/ \mu \mathrm{m}^{2}$. (c) Alveolar wall of lung from patient 6 . Immunogold localization of elastase using rabbit anti-human neutrophil elastase IgG as the primary antibody. Gold at solid arrows. Gold density on elastin $=155$ particles $/ \mu \mathrm{m}^{2}$. Gold density on collagen $(C)=$ eight particles $/ \mu \mathrm{m}^{2}$. (d) Alveolar wall of lung from patient 6 treated as control with rabbit anti-human neutrophil elastase IgG preadsorbed with excess antigen. Gold at solid arrows. Gold density on elastin $=$ six particles $/ \mu \mathrm{m}^{2} . \times 30,000$. 
munogold method. The immunogold method corroborated the immunoperoxidase localization of elastase and removed the ambiguity associated with possible interference of endogenous peroxidase or staining artifacts. Using the immunoperoxidase method, the distribution of elastase on elastin in the lung tissue was estimated morphometrically by determining the relative area of elastin exhibiting elastase-positive reaction product. The results show that the area of elastin identified with elastase reaction product increased with increasing severity of emphysema, as determined by a subjective grading system (Table III), or by mean linear intercept, a more objective grading system (Fig. 8). On the average, sections from lung tissue rated as having mild emphysema had significantly fewer areas of elastin covered with elastase reaction product than sections showing locally severe emphysema (Table III). Thus, a linear correlation between elastase distribution and MLI was found in the lobes of this group

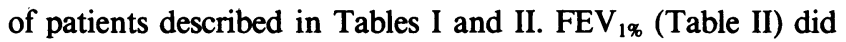
not correlate with smoking history, a not unexpected finding in this small group. There was no correlation between the values of $\mathrm{FEV}_{1 \%}$ and MLI or \% EPGS. This is also not an unexpected result in that histologic evaluation and \% EPGS represented only a small volume of a single lobe and the lesions and elastase concentrations were nonuniformly distributed. As expected, upper lobes tended to show more severe lesions and increased elastase distribution than lower lobes. Although seven of the eight patients were smokers, the special effect of that cigarette smoke on the migration of neutrophils, the release of elastase and the process of elastin damage is not elucidated by these studies. However, several reports suggest a major role for cigarette smoke in these events (26-29).

Although completely "normal" nonsmokers' lungs were not available for these studies, we found that normal lung tissue from patients with histologic evidence of mild emphysema (patients 4 and 8, ๑, Fig. 8) had little or no elastase-positive elastin. All patients in this study had relatively normal pulmonary function even though some of the patients had abnormal histopathology (Figs. 7 and 8). Based upon our initial findings, we anticipate that normal lung tissue from normal nonsmokers lungs would exhibit little or no elastase bound to elastin, similar to what we observed for normal tissue from minimally emphysematous lungs. Lungs from autopsies may provide a large population from which to select normal nonsmokers' lungs and would allow examination of a larger portion of the entire lung. However, complete pulmonary function information on individuals whose tissue may be donated at autopsy may not be available. In addition, it is not known to what extent autolysis could affect the distribution of elastase on the alveolar interstitium. Consequently, surgical specimens were used for our initial studies even though it was clear that normal nonsmoker's lungs would not be available and that it would be difficult to assess the distribution of normal lung tissue in the surgical specimen. Our studies demonstrated that there was a correlation between the local distribution of neutrophil elastase and the local presence of emphysematous change as determined by MLI of the various histologic sections and not with the level of emphysema of the whole lung.

The "protease-protease inhibitor balance" hypothesis proposes that neutrophils can release elastase to the lung interstitium where the elastase, if uninhibited, can bind to and degrade elastin. The present results suggest that elastase released to the interstitium does bind to interstitial elastin as predicted by the proteaseprotease inhibitor balance hypothesis. In addition, the results suggest that elastase is localized and retained in the interstitium in a nonuniform distribution, and may increase episodically as neutrophils migrate and release elastase to the interstitium in response to stimuli such as cigarette smoke. Because very few interstitial neutrophils were observed in the lungs of these patients, even with abundant elastase present, the process of elastin destruction of the lung may continue long after neutrophils have deposited elastase and disappeared from the lung. However, the turnover of elastase in the lung remains unknown. When radiolabeled pancreatic elastase is instilled intratracheally into hamsters $(30)$ or rats $(31)$, the bulk of the enzyme is rapidly cleared, but $1 \%$ of the instilled enzyme is present in the lungs 3-6 d after administration. Similar studies have not been done with neutrophil elastase.

It is likely that neutrophil elastase, possibly released by neutrophils migrating through the alveolar interstitium or penetrating the epithelial or endothelial cell layers, can bind to elastin even in the presence of normal quantities of alpha-1-PI inhibitor. Campbell et al. (32) showed that neutrophils, in close proximity to the connective tissue protein, fibronectin, can release elastase and digest the fibronectin. This proteolysis was incompletely inhibited by alpha-1-PI. Weiss and Regiani (33) showed that neutrophils could degrade a complex subendothelial matrix by releasing elastase when the cells were in close contact with the matrix and this proteolysis was only partially inhibited by large quantities of alpha-1-PI. Using various systems, a number of investigators have shown that once elastase binds to elastin, alpha-1-PI (34), phenylmethane sulfonyl fluoride (35), or the small molecular weight chloromethyl ketone protease inhibitors (36) are only modestly effective in inhibiting proteolysis.

Because of the very tight binding of elastase to elastin, it is likely that the interstitial elastic fiber acts as an affinity matrix and concentrates the enzyme in identifiable locations within the alveolar interstitium. This may explain the infrequent observation of localization of the enzyme on the basement membrane. The use of gold-labeled antibody (Figs. 3-6) allows quantitation of the enzyme, and with increased sensitivity, it may be possible to establish whether or not small amounts of enzyme are preferentially bound to other matrix components in the lungs of smokers. Antigen concentration or reactivity may also be responsible for the lack of any neutrophil elastase labeling in the few interstitial macrophages observed during this study. Although it is well established that alveolar macrophages are capable of receptor-mediated binding and internalization of neutrophil elastase, the macrophages do not bind elastase which has been previously coupled to alpha-1-PI (37). Because the same macrophage receptor may recognize other neutrophil granule proteins (38), release of lactoferrin or cathepsin $\mathrm{G}$ may also reduce the efficacy of elastase uptake by the macrophage. In addition,

Figure 6. (Opposite) Electron micrographs of immunogold localization of neutrophil elastase on purified amorphous human lung elastin exposed to neutrophil elastase. (a) $2.5 \mu \mathrm{g}$ of elastase/mg dry weight elastin. Density of gold particles $=120$ particles $/ \mu \mathrm{m}^{2}$. (b) $25 \mu \mathrm{g}$ elastase $/$ $\mathrm{mg}$ dry weight elastin. Density of gold particles $=1,420 / \mu \mathrm{m}^{2}$. $\times 66,530$

Figure 7. (Opposite) Light micrographs of histologic slides showing the subjective classification of mild $(a)$, moderate $(b)$, and severe $(c)$ emphysema. Asterisks mark enlarged air spaces seen occasionally in mild, more frequently in moderate, and frequently in severe emphysema. Hematoxylin $\&$ eosin stained. $\times 50$. 

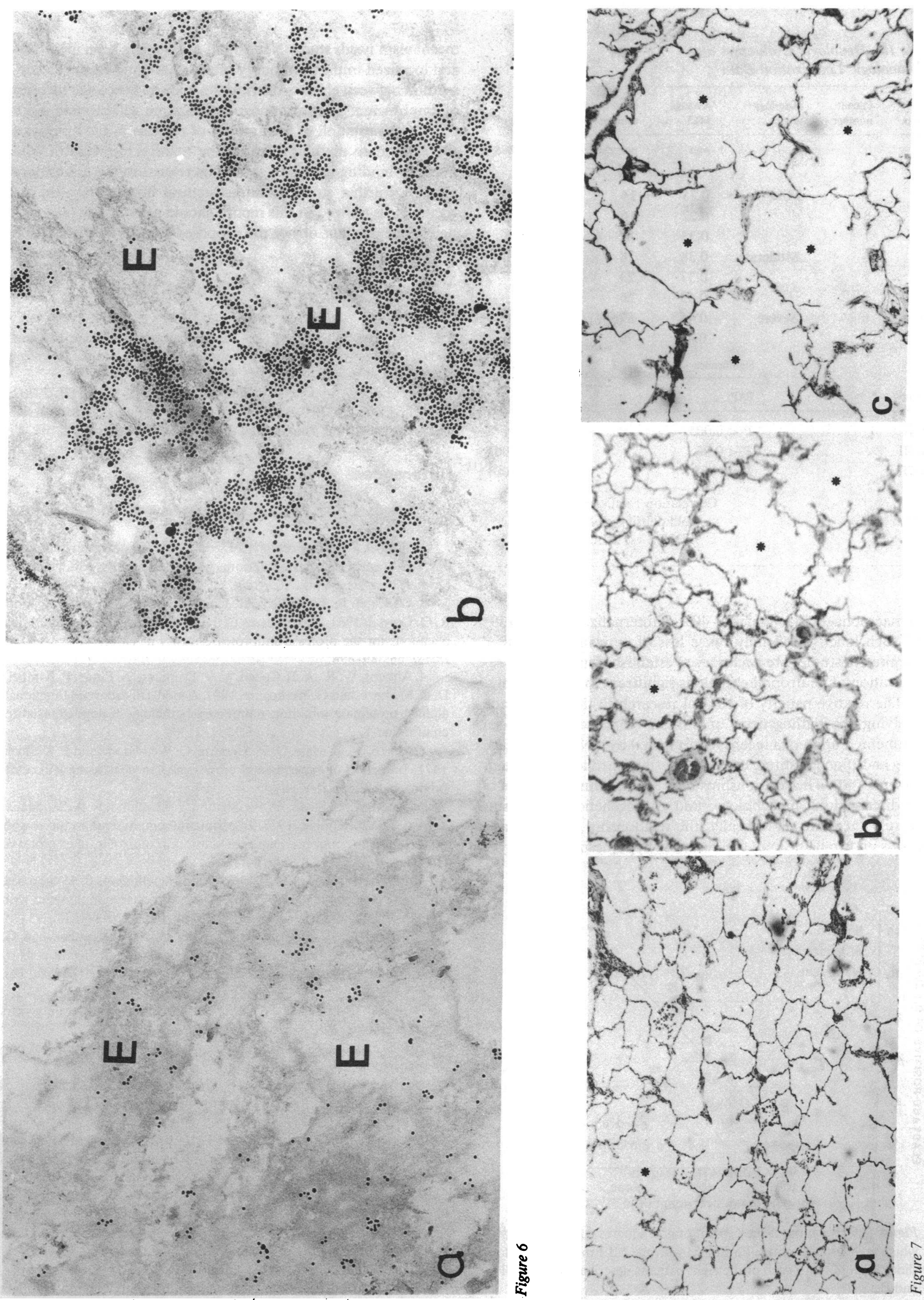
Table III. Histologic Evaluation and Immunologic Localization Data

\begin{tabular}{|c|c|c|c|c|c|}
\hline Group & $\begin{array}{l}\text { Patient } \\
\text { number }\end{array}$ & $\begin{array}{l}\text { Histology } \\
\text { grade }\end{array}$ & $\begin{array}{l}\text { Average } \\
\text { MLI }\end{array}$ & $\begin{array}{l}\text { Gold density } \\
\text { average }\end{array}$ & $\begin{array}{l}\text { PAP } \\
\text { average }\end{array}$ \\
\hline & & & $m m$ & $\begin{array}{l}\text { particles/ } \mu m^{2} \\
\text { elastin }\end{array}$ & \% EPGS \\
\hline \multirow[t]{3}{*}{ I } & 4 & \multirow[t]{3}{*}{ Mild } & 0.25 & 14 & 6 \\
\hline & 8 & & 0.22 & 16 & 9 \\
\hline & 1 & & 0.33 & 11 & 24 \\
\hline \multirow[t]{3}{*}{ II } & 3 & \multirow[t]{2}{*}{ Moderate } & 0.38 & 28 & 26 \\
\hline & 7 & & 0.37 & 23 & 28 \\
\hline & 2 & & 0.43 & 26 & 35 \\
\hline \multirow[t]{3}{*}{ III } & 6 & Severe & 0.46 & 87 & 56 \\
\hline & 5 & & 0.42 & 70 & 54 \\
\hline & & \multicolumn{3}{|c|}{ Significance } & \\
\hline \multicolumn{2}{|l|}{ Group } & \multicolumn{3}{|c|}{ PAP } & Gold \\
\hline \multirow{4}{*}{$\begin{array}{l}\text { I-II } \\
\text { II-III } \\
\text { I-III }\end{array}$} & & \multicolumn{3}{|c|}{$P<0.005$} & $P<0.1$ \\
\hline & & \multicolumn{3}{|c|}{$P<0.05$} & $P<0.001$ \\
\hline & & \multicolumn{3}{|c|}{$P<0.005$} & $P<0.001$ \\
\hline & & \multicolumn{3}{|c|}{$\begin{array}{l}\text { Correlation coefficient } \\
\text { Gold density vs. MLI } \\
\text { \% EPGS vs. MLI }\end{array}$} & $\begin{array}{l}r \\
0.71 \\
0.91\end{array}$ \\
\hline
\end{tabular}

the macrophages inactivate $35-40 \%$ of internalized elastase (39) and such inactivation may cause loss of antigenicity. Further quantitative studies are necessary to establish if macrophage sequestration of neutrophil elastase is significant in human lungs.

The relative paucity of clinical research in the past decade involving the pathogenesis of human emphysema has been commented upon in a recent report issued by a National Heart, Lung and Blood Institute committee established to redefine emphysema (40). The relationships between the clinical aspects of the disease, lung morphology, and pathogenetic mechanisms are still uncertain. Specifically, the relationship between early lesions in smokers and the recently hypothesized elastolytic

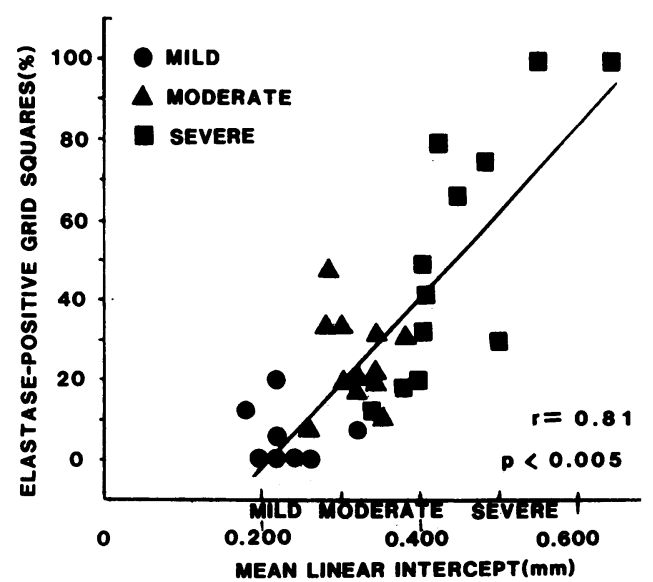

Figure 8. Correlation of the percent of grid squares containing elastin which are elastase-positive (\% EPGS) with the MLI measured on four slides from lungs of each of the eight patients described in Tables I and II. mechanism needs study. Neutrophil elastase has been identified and localized in the pulmonary interstitium, and there is a correlation between elastase distribution and the histologic severity of emphysema. The immunogold technique will provide more precise quantitation of the amount of elastase in the lung than the peroxidase method, because the relative numbers of gold particles binding to elastin should be related to the quantity of elastase. Further study by ultrastructural immunolocalization methods, as utilized in this report, should provide the means to determine whether or not the proposed elastolytic mechanism is valid.

\section{Acknowledgments}

The authors are indebted to Drs. Harry Schwamm, William Manion, and Oleg Guriev for the primary fixation of the lobes and the histologic slides.

This work was supported by grants HL-29734, HL-30446, HL-29702, HL-35264, and 2SO7RR-05874-04 from the U.S. Public Health Service and grant 1604 from The Council for Tobacco Research-USA, Inc.

\section{References}

1. Janoff, A. 1985. Elastases and emphysema. Am. Rev. Respir. Dis. 132:417-433

2. Kimbel, P. 1985. Proteolytic Damage and Emphysema Pathogenesis. In Chronic Obstructive Pulmonary Disease. T. Petty, editor, Marcel Dekker, Inc., New York. 105-127.

3. Gadek, J. E., G. A. Fells, R. L. Zimmerman, S. I. Rennard, and R. G. Crystal. 1981. Antielastases of the human alveolar structures: implications for the protease-antiprotease theory of emphysema. J. Clin. Invest. 68:889-898.

4. Abrams, W. R., A. B. Cohen, V. V. Damiano, A. Eliraz, P. Kimbel, D. R. Meranze, and G. Weinbaum. 1981. A model of decreased functional alpha-1-proteinase inhibitor: pulmonary pathology of dogs exposed to chloramine-T. J. Clin. Invest. 68:1132-1139.

5. Marco, V., B. Mass, D. R. Meranze, G. Weinbaum, and P. Kimbel. 1971. Induction of experimental emphysema in dogs using leukocyte homogenates. Am. Rev. Respir. Dis. 104:595-598.

6. Janoff, A., B. Sloan, G. Weinbaum, V. Damiano, R. A. Sandhaus, J. Elias, and P. Kimbel. 1977. Experimental emphysema induced with purified human neutrophil elastase: tissue localization of instilled protease. Am. Rev. Respir. Dis. 115:461-478.

7. Senior, R. M., H. Tegner, C. Kuhn, K. Ohlsson, B. C. Starcher, and J. A. Pierce. 1977. The induction of pulmonary emphysema with human leukocyte elastase. Am. Rev. Respir. Dis. 116:469-475.

8. Sloan, B., W. R. Abrams, D. R. Meranze, P. Kimbel, and G. Weinbaum. 1981. Emphysema induced in vitro and in vivo in dogs by a purified elastase from homologous leukocytes. Am. Rev. Respir. Dis. 123:295-301.

9. Snider, G. L. 1981. The pathogenesis of emphysema: twenty years of progress. Am. Rev. Respir. Dis. 124:321-324.

10. Dunnil, M. S. 1962. Quantitative methods in the study of pulmonary pathology. Thorax. 17:320-328.

11. McDowell, E. M., and B. F. Trump. 1976. Histological fixatives suitable for diagnostic light and electron microscopy. Arch. Pathol. Lab. Med. 100:405-414.

12. Segal, A., A. Fortunato, and T. Herd. 1980. A rapid single centrifugation step method for the separation of erythrocytes, granulocytes and mononuclear cells on a continuous density gradient of Percoll. $J$. Immunol. Methods. 32:209-214.

13. Watt, S. M., A. W. Burgess, and D. Metcalf. 1979. Isolation and surface labeling of murine polymorphonuclear neutrophils. J. Cell. Physiol. 100:1-21.

14. Damiano, V. V., A. Sandler, W. R. Abrams, D. R. Meranze, A. B. Cohen, P. Kimbel, and G. Weinbaum. 1980. Electron and light 
microscopic studies of the lung of chloramine-T treated dogs. Bull. Eur. Physiopathol. Respir. 16(Suppl.):141-156.

15. Abrams, W. R., and H. L. James. 1979. Dog and human neutrophil elastase: purification and characterization. Fed. Proc. 38:833.

16. Baugh, R. J., and J. Travis. 1976. Human leukocyte granule elastase: rapid isolation and characterization. Biochemistry. 15:836-841.

17. Delshammer, K. M., and K. Ohlsson. 1976. Isolation and partial characterization of elastase from dog granulocytes. Eur. J. Biochem. 69: $125-131$.

18. Ouchterlony, O., and L. A. Nilsson. 1973. Immunodiffusion and immunoelectrophoresis. In Handbook of Experimental Immunology. Vol. I. D. M. Weir, editor. Blackwell Scientific Publications, Oxford. 19.1-19.39.

19. Kucich, U., W. R. Abrams, and H. L. James. 1980. Solid phase immunoassay of dog neutrophil elastase. Anal. Biochem. 109:403-409.

20. Sternberger, L. A. 1971. The unlabeled antibody peroxidase and the immunouranium methods in light and electron microscopy. In: Techniques of Biophysical and Biochemical Morphology, Vol. I. D. Glick and R. M. Rosenbaum, editors. John Wiley \& Sons, New York. 67-87.

21. Horisberger, M. 1979. Evaluation of colloidal gold as a cytochem marker for TEM and SEM. Biol. Cell. 36:253-258.

22. Bainton, D. F., J. L. Ullyot, and M. G. Farquhar. 1971. The development of neutrophilic polymorphonuclear leukocytes in human bone marrow. J. Exp. Med. 134:907-934.

23. Baggiolini, M., V. Bretz, B. Dewald, and M. E. Felgenson. 1978. The polymorphonuclear leukocyte. Agents Actions. 8:3-10.

24. Graham, R. C., and M. J. Karnovsky. 1966. The early stages of absorption of injected horseradish peroxidase in the proximal tubules of mouse kidneys: ultrastructural cytochemistry by a new technique. $J$. Histochem. 14:291-302.

25. Travis, J., and G. S. Salvesen. 1983. Human plasma proteinase inhibitors. Annu. Rev. Biochem. 52:655-709.

26. Cosio, M. G., K. A. Hale, and D. E. Niewoehner. 1980. Morphologic and morphometric effects of prolonged cigarette smoking on the small airways. Am. Rev. Respir. Dis. 122:265-271.

27. Wright, J. L., L. M. Lawson, P. D. Pare, B. J. Wiggs, S. Kennedy, and J. C. Hogg. 1983. Morphology of peripheral airways in current smokers and ex-smokers. Am. Rev. Respir. Dis. 127:474-477.

28. Hoidal, J. R., and D. E. Niewoehner. 1983. Cigarette smoke inhalation potentiates elastase-induced emphysema in hamsters. $\mathbf{A m}$. Rev. Respir. Dis. 126:548-552.
29. Hunninghake, G. W., and R. G. Crystal. 1983. Cigarette smoking and lung destruction: accumulation of neutrophils in the lungs of cigarette smokers. Am. Rev. Respir. Dis. 128:833-836.

30. Stone, P. J., J. D. Calore, G. L. Snider, and C. Franzblau. 1979. The dose-dependent fate of enzymatically active and inactivated tritiated methylated pancreatic elastase administered intratracheally in the hamster. Am. Rev. Respir. Dis. 120:577-587.

31. Sandhaus, R. A., and A. Janoff. 1982. Elastase-induced emphysema: retention of instilled proteinase in the rat. Am. Rev. Respir. Dis. 126:914-920.

32. Campbell, E. J., R. M. Senior, J. A. McDonald, and D. L. Cox. 1982. Proteolysis by neutrophils: relative importance of cell-substrate contact and oxidative inactivation of proteinase inhibitors in vitro. $J$. Clin. Invest. 70:845-852.

33. Weiss, S. J., and S. Regiani. 1984. Neutrophils degrade subendothelial matrices in the presence of alpha-1-proteinase inhibitor. J. Clin. Invest. 73:1297-1303.

34. Reilly, C. F., and J. Travis. 1980. The degradation of human lung elastin by neutrophil proteinases. Biochim. Biophys. Acta. 621:147157.

35. Kuhn, C., W. Engleman, M. Chraplyvy, and B. C. Starcher. 1983. Degradation of elastin in experimental elastase-induced emphysema measured by a radioimmunoassay for desmosine. Exp. Lung Res. 5: 115-123.

36. Powers, J. C. 1983. Synthetic elastase inhibitors: prospects for use in the treatment of emphysema. Am. Rev. Respir. Dis. 127:S54-S58.

37. Campbell, E. S., R. R. White, R. M. Senior, R. J. Rodriguez, and C. Kuhn. 1979. Receptor-mediated binding and internalization of leukocyte elastase by alveolar macrophages in vitro. J. Clin. Invest. 64: 824-833.

38. Campbell, E. J. 1982. Human leukocyte elastase, cathepsin G and lactoferrin: family of neutrophil granule glycoproteins that bind to an alveolar macrophage receptor. Proc. Natl. Acad. Sci. USA. 79:69416945.

39. McGowan, S. E., R. D. Arbeit, P. J. Stone, and G. L. Snider. 1983. A comparison of the binding and fate of internalized neutrophil elastase in human monocytes and alveolar macrophages. Am. Rev. Respir. Dis. 128:688-694.

40. Snider, G. L., J. Kleinerman, W. M. Thurlbeck, and Z. H. Bengali. 1985. The definition of emphysema. Am. Rev. Respir. Dis. 132:182185. 